\title{
All-electrical generation of spin-polarized currents in quantum spin Hall insulators
}

\author{
L. L. Tao, ${ }^{1}$ K. T. Cheung, ${ }^{1}$ L. Zhang, ${ }^{2,1,3}$ and J. Wang ${ }^{1, *}$ \\ ${ }^{1}$ Department of Physics and the Center of Theoretical and Computational Physics, \\ The University of Hong Kong, Hong Kong, China \\ ${ }^{2}$ State Key Laboratory of Quantum Optics and Quantum Optics Devices, Institute of Laser Spectroscopy, \\ Shanxi University, Taiyuan 030006, China \\ ${ }^{3}$ Collaborative Innovation Center of Extreme Optics, Shanxi University, Taiyuan 030006, China \\ (Received 2 September 2016; revised manuscript received 16 November 2016; published 24 March 2017)
}

\begin{abstract}
The control and generation of spin-polarized current (SPC) without magnetic materials and an external magnetic field is a big challenge in spintronics and normally requires a spin-flip mechanism. In this Rapid Communication, we show the theoretical discovery of all-electrical generation of SPC without relying on spin-flip spin-orbit coupling (SOC). We find that the SPC can be produced as long as an energy-dependent phase difference between the spin up and down electrons can be established. We verify this through quantum transport calculations on a gated stanene zigzag nanoribbon, which is a quantum spin Hall (QSH) insulator. Our calculations indicate that the transient current as well as ac conductance are significantly spin polarized, which results from the genetic phase difference between spin up and down electrons after traversing the system. Our results are robust against edge imperfections and generally valid for other QSH insulators, such as silicene and germanene, etc. These findings establish a different route for generating SPCs by purely electrical means and open the door for interesting applications of semiconductor spintronics.
\end{abstract}

DOI: 10.1103/PhysRevB.95.121407

Introduction. Spintronics exploits electron spin degrees of freedom which display various fascinating physics and bring many promising technological applications $[1,2]$. One of the central issues of spintronics is the control and generation of spin-polarized currents (SPCs). Recently, the generation and detection of SPCs at the nanoscale by purely electrical means has attracted great attention since this is the key step towards developing semiconductor spintronic devices [3-5]. The conventional way of producing SPCs requires the application of magnetic fields [6,7] or ferromagnetic materials $[8,9]$, which is conceptually different from the field-effect devices and difficult to integrate into existing semiconductor devices [10]. Thus it would be highly desirable to generate SPCs by purely electrical means. Naturally, the system with spin-orbit coupling (SOC) in a particular Rashba SOC is a promising candidate to achieve those objectives by exploiting the link between an electron spin and its space motion [11]. Indeed, it has been shown theoretically that SPCs can be produced by electrical means due to Rashba SOC in several systems, such as two-dimensional electron gas [12], graphene nanoribbons [13], and carbon nanotubes [14]. Being a spin-flip SOC, Rashba SOC has been extensively investigated for the generation of nonequilibrium spin polarization $[15,16]$, spin-polarized current $[13,14]$, and pure spin current (spin Hall effect) [17]. However, spin-flip SOC is detrimental to the spin lifetime, as expected from the spin-flip scattering. A different route to generate SPC without spin-flip SOC is clearly worth exploring both for scientific interest and practical applications. Since the generation of SPC normally requires a spin-flip mechanism, a natural question arises: Is it possible to induce a SPC using electrical means in the absence of spin-flip SOC?

\footnotetext{
*jianwang@hku.hk
}

Partial answers are available from previous studies $[4,5,18,19]$. It was demonstrated theoretically that opposite spin accumulations on the transverse edges can be induced by a strong lateral SOC (intrinsic SOC that conserves spin) in the two-dimensional electron gas [18], although a SPC cannot be induced. Experimentally, Debray et al. [4] found a conductance plateau around half conductance quanta in a quantum point contact device with a large lateral SOC driven by an asymmetry confining potential. However, it was found $[4,5,19]$ that in order to reproduce the plateau of half conductance quanta it is essential to consider a strong $e-e$ interaction acting as a spin-dependent potential, implying that an additional magnetic-field-like term was required. Thus the time-reversal symmetry is broken naturally.

In this Rapid Communication, we propose a conceptually different way of generating SPCs using the intrinsic SOC, that conserves spin, in a series of quantum spin Hall (QSH) insulators, such as silicene [20], germanene [21], and stanene [22], whose band gaps are very large (ranging 1-100 meV) $[23,24]$ in comparison with graphene $\left(10^{-3} \mathrm{meV}\right)[25]$. QSH insulators are a new state of quantum matter characterized by an insulating bulk and topologically protected gapless edge states [26,27]. To produce SPCs using electrical means it seems that one must flip the spin to make the transmission probabilities imbalance between spin up and down channels. The working principle of our proposal is to tune the relative phase between the wave function of different spins that causes a time delay between spin up and down electrons traversing the system, which gives rise to SPCs in the ac signals. Specifically, this is achieved by applying a pulse or ac source-drain voltage on the central-region-gated QSH insulator zigzag nanoribbons without any further decoration or doping. Without losing generality, we consider a practical QSH insulator device model, i.e., stanene zigzag nanoribbons, as shown in Fig. 1. We emphasize that the analysis and discussion presented here are very general and are applicable to silicene and germanene as 

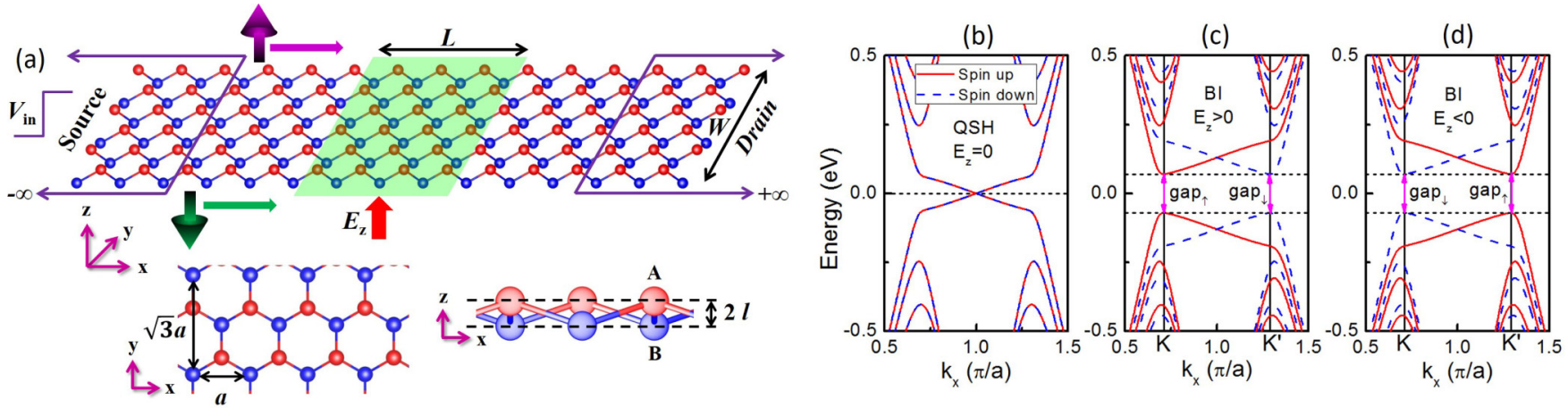

FIG. 1. (a) Schematic plot of a zigzag nanoribbon device with different views. Here, $E_{z}$ is the external electric field generated by the back-gate voltage. The source and drain electrodes extend to $x=\mp \infty$, as shown by the left/right pointing arrows. $V_{\text {in }}$ is the input pulse voltage. $a$ is the lattice constant. $l$ is the buckling height. $W$ (unit $\sqrt{3} a$ ) and $L$ (unit $a$ ) denote the width and the length of the gated region, respectively. (b) -(d) Band structures for the nanoribbon with $W=12$. (b) QSH insulator phase with $E_{z}=0$. Trivial BI phase with (c) $l E_{z}=0.1 h_{0}\left(E_{z}>0\right)$ and (d) $l E_{z}=-0.1 h_{0}\left(E_{z}<0\right) . K(0.71)$ and $K^{\prime}(1.29)$ denote the position of the conduction band minimum (or valence band maximum) for different spins. Gap (gap $_{\downarrow}$ ) denotes the band gap for spin up (spin down).

well. We also expect those findings to be a general feature of open quantum systems as long as an energy-dependent phase difference between spin up and down electrons can be established by electrical means.

The model and theoretical formalism. The tight-binding Hamiltonian for describing the QSH insulator nanoribbon is given by $[7,24,28]$,

$$
\begin{aligned}
H= & -h_{0} \sum_{\langle i, j\rangle \alpha} c_{i \alpha}^{\dagger} c_{j \alpha}+i \frac{\lambda \mathrm{SO}}{3 \sqrt{3}} \sum_{\langle\langle i, j\rangle\rangle \alpha, \beta} v_{i j} c_{i \alpha}^{\dagger} \sigma_{z}^{\alpha \beta} c_{j \beta} \\
& +l E_{z} \sum_{i \alpha} \xi_{i} c_{i \alpha}^{\dagger} c_{i \alpha} .
\end{aligned}
$$

The first term is the hopping term, where $c_{i \alpha}^{\dagger}\left(c_{j \alpha}\right)$ is an electron creation (annihilation) operator at site $i(j)$ with $\operatorname{spin} \alpha=\uparrow, \downarrow$, $h_{0}$ is the hopping energy, and $\langle i, j\rangle$ denotes the sum over the nearest-neighbor sites. The second term represents the intrinsic SOC with strength $\lambda_{\mathrm{SO}},\langle\langle i, j\rangle\rangle$ denotes the sum over the nextnearest-neighbor sites, $\sigma_{z}$ is the $z$-component Pauli matrix, and $v_{i j}=+1(-1)$ if the hopping is anticlockwise (clockwise) with respect to the $z$ axis [26]. The third term arises from the applied electric field $E_{z}, \xi_{i}= \pm 1$ for $i=A, B$ sites, and $l$ is the buckling height. The following tight-binding parameters for stanane nanoribbon are used: $h_{0}=1.3 \mathrm{eV}, \lambda_{\mathrm{SO}}=0.1 \mathrm{eV}$, $a=4.70 \AA$, and $l=0.40 \AA$ [24].

For the transient current calculation, the time-dependent terminal current $I_{\alpha}(t)$ of lead $\alpha$ is given by [29]

$$
I_{\alpha}(t)=2 \operatorname{Re} \operatorname{Tr}\left[\bar{\Gamma}_{\alpha} H_{c c} G_{c c}^{<}(t, t) \bar{\Gamma}_{\alpha}-i \bar{\Gamma}_{\alpha} \partial_{t} G_{c c}^{<}(t, t) \bar{\Gamma}_{\alpha}\right],
$$

where $\bar{\Gamma}_{\alpha}$ is an auxiliary projection matrix, and $G_{c c}^{<}(t, t)$ and $H_{c c}$ are the time-dependent lesser Green's function and the Hamiltonian of the central scattering region, respectively. We consider both upward and downward voltage pulses [29,30]. In the ac regime, the ac current consists of a particle current and displacement current [31-33], namely, the total dynamic conductance $G_{L R}=G_{L R}^{c}+G_{L R}^{d}$. Particle conductance $G_{L R}^{c}$ can be expressed as [31]

$$
G_{L R}^{c}=-\int \frac{d E}{2 \pi} \frac{f-\bar{f}}{\omega} \operatorname{Tr}\left[s_{L R}^{\dagger}(E) s_{L R}(E+\omega)\right],
$$

where $f$ is the Fermi function, $s_{L R}$ the scattering matrix, $\omega$ the frequency, and $\bar{f} \equiv f(E+\omega)$. The complicated expression of displacement conductance $G_{L R}^{d}$ is given in the Supplemental Material [34].

Results and discussion. We first analyze the electronic structure of the stanene zigzag nanoribbon. Previous work shows that the stanene as well as silicene and germanene reveal rich topological phases driven by $E_{z}[6,24]$, which is distinct from graphene. Figures 1(b)-1(d) show the band structures for the nanoribbon with $E_{z}=0, E_{z}>0$, and $E_{z}<0$. For $E_{z}=0$ shown in Fig. 1(b), the nanoribbon is in the QSH insulator phase, characterized by the gapless edge states and the band is spin degenerate. For $E_{z}>0$ (larger than the critical field [24]) shown in Fig. 1(c), a finite gap is opened up and the nanoribbon is driven into a trivial band insulator (BI) phase. More interestingly, the spin degeneracy is lifted due to the combined effect of the inversion symmetry breaking by $E_{z}$ and the intrinsic SOC, which is the key for the generation of SPCs. For $E_{z}<0$ (same field magnitude with $E_{z}>0$ ) shown in Fig. 1(d), comparing with Fig. 1(c), we see that the reversal of $E_{z}$ interchanges the role of spin up and down electrons, as expected from the potential interchange between $A$ and $B$ sites. Note that with $E_{z}$ turned on, the spin component $s_{z}$ is still a good quantum number, as is evident from $\left[s_{z}, H\right]=0$.

We now connect our system with two leads consisting of a stanene nanoribbon and study the transport behaviors. Figures 2(a)-2(c) show the spin-resolved transient current $I_{\sigma}(t)$ and spin-polarized current $P(t)=I_{\uparrow}(t)-I_{\downarrow}(t)$ for an upward voltage pulse. Overall, $I_{\sigma}(t)$ evolves in three distinct regimes: an initial strong oscillating regime, followed by a steady increase, and finally approaching the dc steady state. For the central region in the QSH insulator phase [see Fig. 2(a)], the transient current is spin degenerate at all times. In contrast, for the central region in the BI phase induced by $E_{z}$ [see Figs. 2(b) and $2(\mathrm{c})]$, the current is strongly spin polarized before reaching the steady state, as shown explicitly in the inset. Therefore, the generation of SPC can be realized in the time window away from the steady state. Interestingly, the characteristic time reaching the steady state is clearly different for spin up and down electrons, namely, $\tau_{\uparrow}>\tau_{\downarrow}\left[E_{z}>0\right.$, 

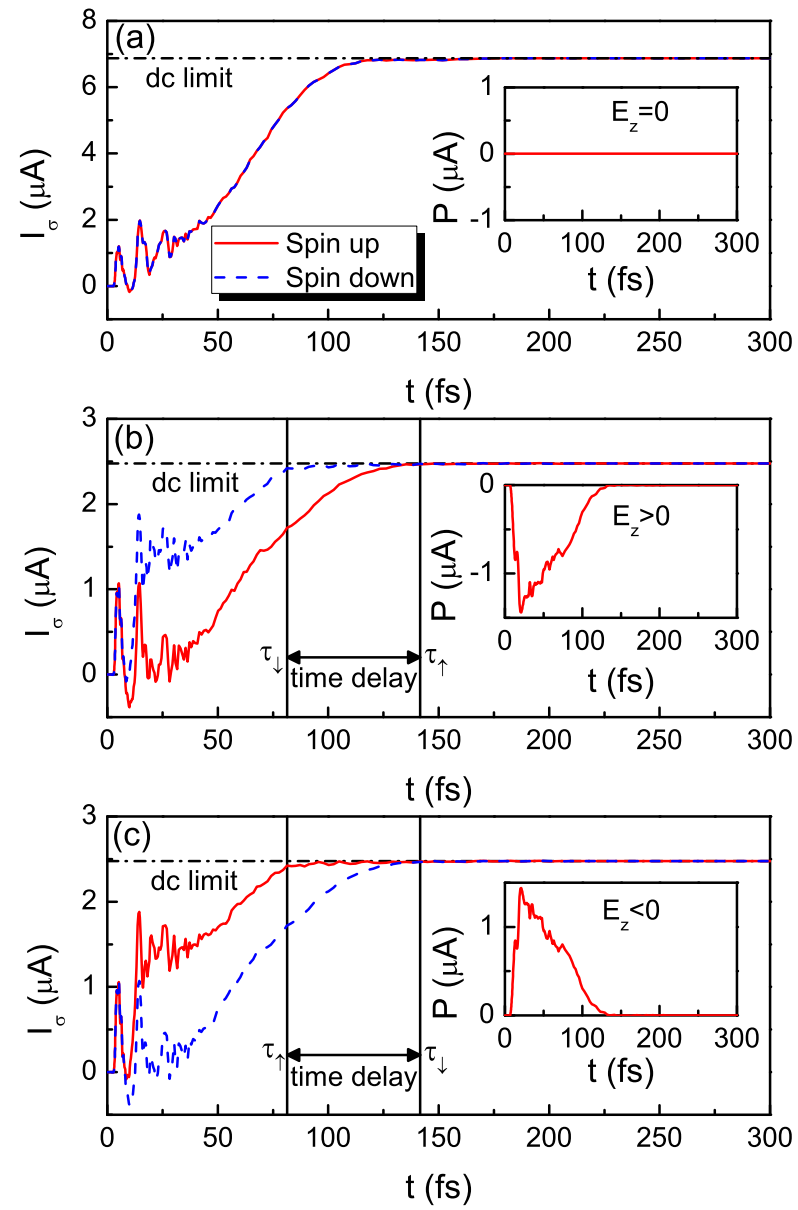

FIG. 2. The transient current $I_{\sigma}(t)(\sigma=\uparrow$ for spin up, $\downarrow$ for spin down) through the nanoribbon in response to a upward step pulse. $W=12, L=16$, and $V_{b}(t)=0.2 \theta(t) \mathrm{V}$. (a) $E_{z}=0$, (b) $l E_{z}=0.1 h_{0}$ $\left(E_{z}>0\right)$, and (c) $l E_{z}=-0.1 h_{0}\left(E_{z}<0\right)$. Inset: The spin-polarized current defined as $P(t)=I_{\uparrow}(t)-I_{\downarrow}(t) . \tau_{\uparrow}\left(\tau_{\downarrow}\right)$ denotes roughly the characteristic time for the spin up (down) electron to reach the steady state. The short-dashed lines that denote the dc current for spin up/down are calculated by using the Landauer-Büttiker formula.

Fig. 2(b)] or $\tau_{\uparrow}<\tau_{\downarrow}$ [ $E_{z}<0$, Fig. 2(c)], showing a time delay between spin up and down electrons. As we shall see later, this time delay is due to the generic phase difference between spin up and down electrons traversing the system. Comparing Fig. 2(c) with Fig. 2(b), it is clear that the sign of $P$ can be changed simply by a reversal of $E_{z}$, as can be inferred from the band structures in Figs. 1(c) and 1(d). We also calculated the transient current for the nanoribbons with different lengths $L$ (barrier thickness), as shown in Fig. S3(a) in the Supplemental Material. Qualitatively, they all display fairly similar features as compared to the case of $L=16$. Moreover, the characteristic time $\tau_{\uparrow}\left(\tau_{\downarrow}\right)$ scales almost linearly with the barrier thickness [see Fig. S3(b)], however, their slopes are different, which results in a linearly increase of time delay with barrier thickness. The pulse amplitude dependency of SPC is given in Fig. S4(b). It is seen that the magnitude of SPC increases monotonously with increasing pulse amplitude, while the time window producing the SPC does not change much. On the other hand, as shown in Fig. S2, we confirmed
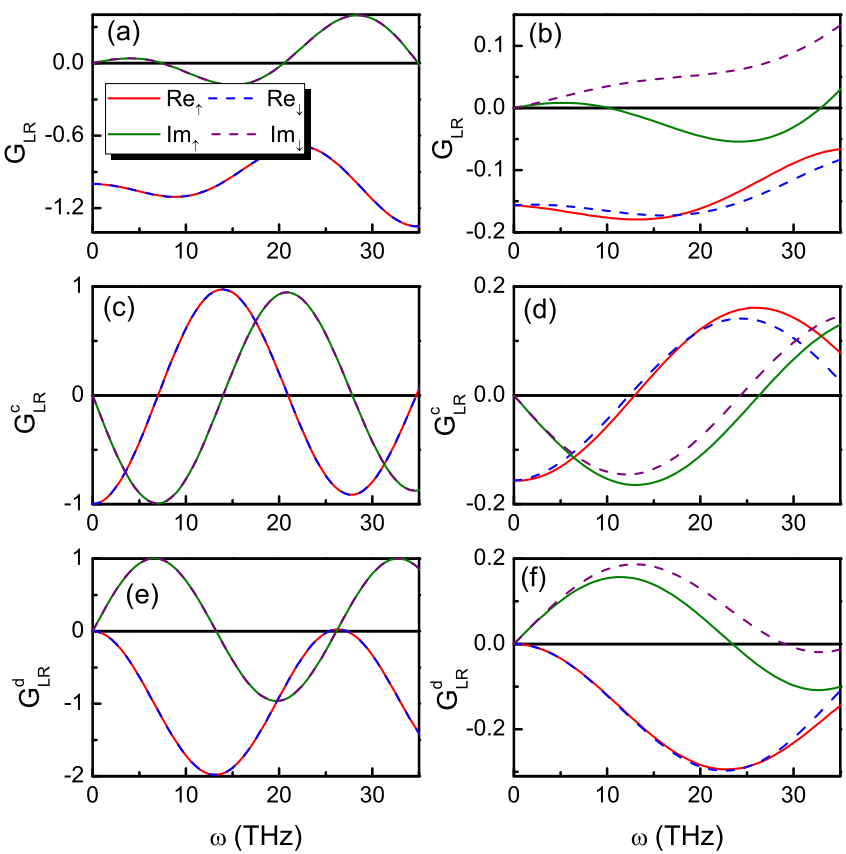

FIG. 3. Dynamic conductance (in units of $e^{2} / h$ ) as a function of frequency $\omega$. (a), (b) The total dynamic conductance $G_{L R}$. (c), (d) The dynamic conductance $G_{L R}^{c}$ contributed by the particle current. (e), (f) The dynamic conductance $G_{L R}^{d}$ contributed by the displacement current. (a), (c), (e) and (b), (d), (f) show the results for the nanoribbon without and with $E_{z}$ in the central region, respectively. Re: real part; Im: imaginary part. Parameters $W, L$, and $E_{z}>0$ are the same as those given in the caption of Fig. 2.

numerically that the SPC also occurs in the transient regime for a downward voltage pulse. This indicates that SPC can be generated for a periodic train of pulses [35]. Note that previous work demonstrated that a SPC can be produced from the time-dependent Rashba SOC generated by a time-dependent gate voltage [36-38]. Its physical mechanism generating SPC is distinctly different from our work, in which the Rashba SOC is absent.

To understand the physics behind the generation of SPC in the transient regime, we study the dynamical conductance $G_{\alpha \beta}(\omega)(\alpha, \beta=L, R)$ as a function of frequency $\omega$ for a sinusoidal bias in the linear response regime since a train of bias pulse can be expanded in terms of simple harmonics with basic frequency. In Fig. 3, we show the frequency dependency of $G_{L R}(\omega)$. For the central region in the QSH insulator phase shown in Fig. 3(a), $G_{L R}(\omega)$ are spin degenerate and exhibit oscillation features due to the interplay of the particle current and displacement current, as evident from Figs. 3(c) and 3(e). The particle current $G_{L R}^{c}$ is found to be $G_{L R}^{c}(\omega)=-e^{i 2 \pi \frac{\omega}{E_{0}}}$ with $E_{0}=17.8 \mathrm{meV}$, which can be understood from the scattering matrix $s_{L R}(E)$ in Fig. 4(a) which relates $G_{L R}^{c}$ through Eq. (3). Analytically, we find $s_{L R}(E)=-e^{i 2 \pi \frac{E}{E_{0}}}$ near the Fermi energy (see the Supplemental Material for the proof of this formula), $E_{0}=2 \pi v_{F} / d$, where $v_{F}$ is the Fermi velocity of edge states and $d$ is the realistic thickness [39]. Note that the energy dependence of $s_{L R}$ is the generic feature of a massless Dirac fermion. Using $v_{F}$ determined from the band dispersion 

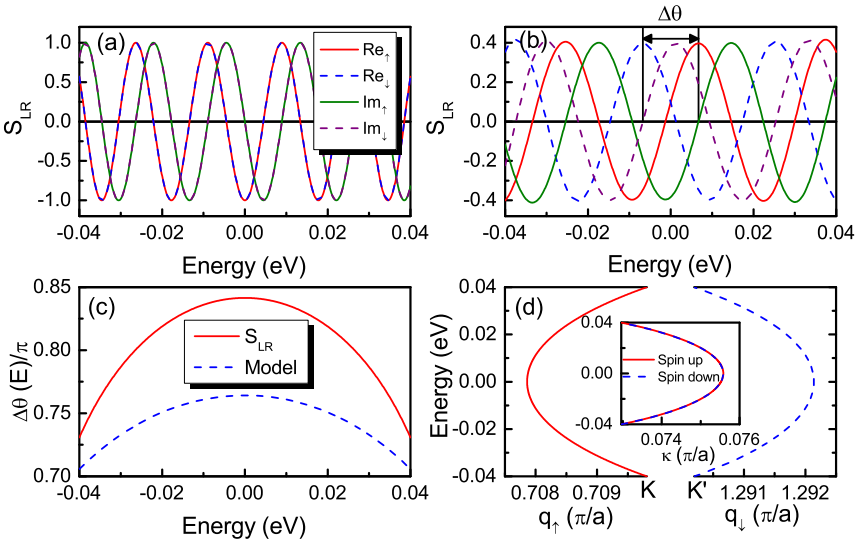

FIG. 4. The scattering matrix $s_{L R}$ as a function of energy for (a) QSH insulator phase and (b) BI phase in the central region. (c) The phase difference $\Delta \theta=\theta_{\downarrow}-\theta_{\uparrow}$ as a function of energy when the central region is in the BI phase. $S_{L R}: \Delta \theta(E)$ is determined from the scattering matrix. Model: $\Delta \theta(E)$ is estimated from $\Delta \theta(E)=$ $\left[q_{\downarrow}(E)-q_{\uparrow}(E)\right] d$. Parameters $W, L$, and $E_{z}>0$ are the same as those given in the caption of Fig. 2. (d) The complex band structure for the nanoribbon in the BI phase. $q$ and $\kappa$ (inset) correspond to the real and imaginary components of wave vector $k_{x}=q+i \kappa$, respectively.

in Fig. 1(b), we find $E_{0}=17.3 \mathrm{meV}$, which agrees well with the numerical result, $E_{0}=17.8 \mathrm{meV}$.

For the central region in the BI phase shown in Figs. 3(b), 3(d), and 3(f), the most striking observation is that the spin degeneracy of dynamic conductance is lifted. The spin-splitting $G_{L R}^{c}$ can be understood from $s_{L R}(E)$, as shown in Fig. 4(b). It is clearly seen that there is a phase difference $\Delta \theta(E)$ for $s_{L R}(E)$ between spin up and down electrons. Importantly, this phase difference has to be energy dependent in order to lift the spin degeneracy, as seen from Eq. (3). From Fig. 4(c), we find that $\Delta \theta(E)$ is indeed energy dependent, which is responsible for spin-splitting $G_{L R}^{c}$.

The observed $\Delta \theta(E)$ can be understood from the physics of one-dimensional quantum tunneling. In contrast to the conventional quantum tunneling picture where the incident electron decays exponentially inside the barrier, the incident edge state (Dirac fermion) near the Fermi energy decays in an oscillatory fashion as $e^{-\kappa x} e^{i q x}$ through the barrier (the gap region), so that an extra phase $q d$ is acquired after the tunneling. This unusual feature is confirmed from the complex band structure as shown in Fig. 4(d), from which the complex wave vector $k_{x}=q+i \kappa$ can be determined for spin up and down electrons. The complex band structure that extends the usual band structure to include complex Bloch vectors is very useful to analyze the quantum transport properties [40-42]. The behavior of the oscillatory decay of edge/surface states was previously reported in the QSH insulator of the Bernevig, Hughes, and Zhang model [43,44] and also in the three-dimensional (3D) topological insulator $\mathrm{Bi}_{2} \mathrm{Se}_{3}$ [45]. Note that the position of the conduction band minimum (or valence band maximum) [see Fig. 1(c)] for the spin up (down) electron is located near the $K\left(K^{\prime}\right)$ point, giving rise to a different $q_{\uparrow}$ or $q_{\downarrow}$ while $\kappa$ is spin degenerate due to the time-reversal symmetry [inset of Fig. 4(d)]. In other words, the electron with different spins acquires different phases after the tunneling with the phase difference $\Delta \theta(E)=$ $\left[q_{\downarrow}(E)-q_{\uparrow}(E)\right] d$, where $q_{\downarrow}$ and $q_{\uparrow}$ are around the $K^{\prime}$ and $K$ point, respectively. The comparison between the model and numerical results is shown in Fig. 4(c). We see that our simple one-dimensional model captured essential physics. Since the transmission coefficient in the first subband $T(E)$ depends weakly on energy [see Fig. S1(b)], the phase accumulated after the tunneling is related to the tunneling time defined as $\tau_{t} \equiv h \operatorname{Im}\left(s_{L R} \partial_{E} s_{L R}^{\dagger}\right) / T(E)=h \partial_{E} \theta(E)$ [46], suggesting a time delay $t_{0}=h \partial_{E} \Delta \theta(E)=h\left(\partial q_{\uparrow}-\partial q_{\downarrow}\right) d$ between spin up and down electrons. Indeed, from Fig. S3(b), we see that the time delay $t_{0}$ increases linearly with the barrier thickness. Now the physics becomes transparent. The spin up electron passing through the energy gap of the BI region experiences a time delay relative to the spin down electron. While this does not change the spin polarization of the dc current since it is time independent, the spin polarization of any ac transport properties will be affected, as shown above. We conclude that the SPC arises from the combined effect of $E_{z}$ and the ac field: (1) $E_{z}$ induces an energy-dependent phase difference between spin up and down electrons, resulting in a time delay between them; (2) this time delay can only be manifested through the inelastic scattering [integrand of Eq. (3)] due to the ac field.

It is known that the local defects, in particular, the atomic vacancy, usually exist at the edges of two-dimensional (2D) honeycomb structures $[47,48]$. To explore the effect of vacancy, we calculated the transient current for the nanorribbon with different edge-vacancy configurations. From Fig. S5(a), the edge vacancies modify slightly the magnitude of the currents in the steady state (dc limit), while the induced SPCs in the transient regime do not change much. In addition, these vacancies have little effect on the conductance, especially in the lower subbands [see Fig. S5(b)].

Conclusion. We have proposed a way of generating a SPC in a family of QSH insulators, such as stanene, silicene, and germanene, etc., zigzag nanoribbons using electrical means. Conceptually different from the conventional all-electrical approaches in producing a SPC $[4,5,49]$, a spin-flip SOC is not required here. The SPC is generated by tuning the phase difference between spin up and down electrons induced by a gate voltage. Considering the experimental feasibility, the bulk band gap of stanene was predicted to be about $0.3 \mathrm{eV}$ [50], which lies above room temperature. On the other hand, our results also suggest an all-electrical way to detect the edge states of QSH insulators.

Acknowledgments. L.L.T. would like to thank Zhizhou Yu and Yanxia Xing for useful discussions. This work is supported by GRF (Grant No. 17311116), University Grant Council of Hong Kong (Contract No. AoE/P-04/08), and the National Natural Science Foundation of China (Grant No. 11374246).
[1] I. Žutić, J. Fabian, and S. Das Sarma, Rev. Mod. Phys. 76, 323 (2004).
[2] D. Pesin and A. H. MacDonald, Nat. Mater. 11, 409 (2012). 
[3] R. Crook, J. Prance, K. J. Thomas, S. J. Chorley, I. Farrer, D. A. Ritchie, M. Pepper, and C. G. Smith, Science 312, 1359 (2006).

[4] P. Debray, S. M. S. Rahman, J. Wan, R. S. Newrock, M. Cahay, A. T. Ngo, S. E. Ulloa, S. T. Herbert, M. Muhammad, and M. Johnson, Nat. Nanotechol. 4, 759 (2009).

[5] P. Chuang, S. C. Ho, L. W. Smith, F. Sfigakis, M. Pepper, C. H. Chen, J. C. Fan, J. P. Griffiths, I. Farrer, H. E. Beere et al., Nat. Nanotechol. 10, 35 (2015).

[6] W. F. Tsai, C. Y. Huang, T. R. Chang, H. Lin, H. T. Jeng, and A. Bansil, Nat. Commun. 4, 1500 (2013).

[7] Kh. Shakouri, H. Simchi, M. Esmaeilzadeh, H. Mazidabadi, and F. M. Peeters, Phys. Rev. B 92, 035413 (2015).

[8] S. Parkin, MRS Bull. 31, 389 (2006).

[9] J. Tang and K. L. Wang, Nanoscale 7, 4325 (2015).

[10] D. D. Awschalom and M. E. Flatte, Nat. Phys. 3, 153 (2007).

[11] A. Manchon, H. C. Koo, J. Nitta, S. M. Frolov, and R. A. Duine, Nat. Mater. 14, 871 (2015).

[12] C. S. Li, Y. J. Yu, Y. D. Wei, and J. Wang, Phys. Rev. B 75, 035312 (2007).

[13] L. Chico, A. Latgé, and L. Brey, Phys. Chem. Chem. Phys. 17, 16469 (2015).

[14] H. Santos, A. Latgé, J. E. Alvarellos, and L. Chico, Phys. Rev. B 93, 165424 (2016).

[15] A. Manchon and S. Zhang, Phys. Rev. B 78, 212405 (2008).

[16] C. H. Li, O. M. J. vant Erve, J. T. Robinson, Y. Liu, L. Li, and B. T. Jonker, Nat. Nanotechol. 9, 218 (2014).

[17] J. Sinova, S. O. Valenzuela, J. Wunderlich, C. H. Back, and T. Jungwirth, Rev. Mod. Phys. 87, 1213 (2015).

[18] Y. X. Xing, Q. F. Sun, L. Tang, and J. P. Hu, Phys. Rev. B 74, 155313 (2006).

[19] A. T. Ngo, P. Debray, and S. E. Ulloa, Phys. Rev. B 81, 115328 (2010).

[20] P. Vogt, P. De Padova, C. Quaresima, J. Avila, E. Frantzeskakis, M. C. Asensio, A. Resta, B. Ealet, and G. Le Lay, Phys. Rev. Lett. 108, 155501 (2012).

[21] M. E. Dávila, L. Xian, S. Cahangirov, A. Rubio, and G. Le Lay, New J. Phys. 16, 095002 (2014).

[22] F. Zhu, W. Chen, Y. Xu, C. Gao, D. Guan, C. Liu, D. Qian, S. C. Zhang, and J. Jia, Nat. Mater. 14, 1020 (2015).

[23] C. C. Liu, H. Jiang, and Y. Yao, Phys. Rev. B 84, 195430 (2011).

[24] M. Ezawa, J. Phys. Soc. Jpn. 84, 121003 (2015).

[25] Y. Yao, F. Ye, X. L. Qi, S. C. Zhang, and Z. Fang, Phys. Rev. B 75, 041401 (2007).

[26] C. L. Kane and E. J. Mele, Phys. Rev. Lett. 95, 226801 (2005).

[27] B. A. Bernevig and S. C. Zhang, Phys. Rev. Lett. 96, 106802 (2006).

[28] S. Rachel and M. Ezawa, Phys. Rev. B 89, 195303 (2014).

[29] L. Zhang, J. Chen, and J. Wang, Phys. Rev. B 87, 205401 (2013).
[30] K. T. Cheung, B. Fu, Z. Yu, and J. Wang, Phys. Rev. B 95, 125422 (2017)

[31] M. Büttiker, A. Prêtre, and H. Thomas, Phys. Rev. Lett. 70, 4114 (1993).

[32] B. Wang, J. Wang, and H. Guo, Phys. Rev. Lett. 82, 398 (1999).

[33] Y. Wei and J. Wang, Phys. Rev. B 79, 195315 (2009).

[34] See Supplemental Material at http://link.aps.org/supplemental/ 10.1103/PhysRevB.95.121407 for additional results of the transient current in response to a downward pulse, barrier length, and pulse-amplitude-dependent transient currents, effect of edge vacancies on the transient currents, and technical details, which includes Refs. [29,30,32,33,42].

[35] Note that the transient current under a periodic voltage pulse is not studied in this work. Consequently, the effect of pulse shape and duration cannot be investigated directly. However, the essential physics discussed in this work is not affected by the pulse shape or duration.

[36] A. G. Mal'shukov, C. S. Tang, C. S. Chu, and K. A. Chao, Phys. Rev. B 68, 233307 (2003).

[37] C. S. Tang, A. G. Mal'shukov, and K. A. Chao, Phys. Rev. B 71, 195314 (2005).

[38] C. H. Lin, C. S. Tang, and Y. C. Chang, Phys. Rev. B 78, 245312 (2008).

[39] In our calculations, $L$ denotes the number of layers being gated by $E_{z}$. The realistic thickness $d$ (unit $a$ ) of the device is $d=$ $L+12-1$ (the buffer layer is fixed as 6) for the central layer in the QSH insulator phase, while it is $d=L-1$ for the central layer in the BI phase.

[40] L. L. Tao, S. H. Liang, D. P. Liu, and X. F. Han, J. Appl. Phys. 114, 213906 (2013).

[41] L. L. Tao, D. P. Liu, S. H. Liang, X. F. Han, and H. Guo, Europhys. Lett. 105, 58003 (2014).

[42] Y. C. Chang, Phys. Rev. B 25, 605 (1982).

[43] X. Dang, J. D. Burton, A. Kalitsov, J. P. Velev, and E. Y. Tsymbal, Phys. Rev. B 90, 155307 (2014).

[44] B. A. Bernevig, T. L. Hughes, and S. C. Zhang, Science 314, 1757 (2006).

[45] J. Betancourt, S. Li, X. Dang, J. D. Burton, E. Y. Tsymbal, and J. P. Velev, J. Phys.: Condens. Matter 28, 395501 (2016).

[46] E. H. Hauge and J. A. Stovneng, Rev. Mod. Phys. 61, 917 (1989).

[47] Y. Niimi, T. Matsui, H. Kambara, K. Tagami, M. Tsukada, and H. Fukuyama, Phys. Rev. B 73, 085421 (2006).

[48] Y. Kobayashi, K. I. Fukui, T. Enoki, and K. Kusakabe, Phys. Rev. B 73, 125415 (2006).

[49] F. Dolcini, R. C. Iotti, A. Montorsi, and F. Rossi, Phys. Rev. B 94, 165412 (2016).

[50] Y. Xu, B. Yan, H. J. Zhang, J. Wang, G. Xu, P. Tang, W. Duan, and S. C. Zhang, Phys. Rev. Lett. 111, 136804 (2013). 